\title{
Light-Echo Spectrum Reveals the Type of Tycho Brahe's 1572 Supernova
}

\author{
T. Usuda ${ }^{1}$, O. Krause ${ }^{2}$, M. Tanaka ${ }^{3}$, T. Hattori ${ }^{1}$, \\ M. Goto ${ }^{2}$, S. M. Birkmann ${ }^{2}$, and K. Nomoto ${ }^{3}$ \\ ${ }^{1}$ SUBARU Telescope, National Astronomical Observatory of Japan, 650 North A'ohoku Place, \\ Hilo, Hawaii, USA \\ email: usuda@subaru.naoj.org \\ ${ }^{2}$ Max-Planck-Institut für Astronomie, Königstuhl 17, 69117 Heidelberg, Germany \\ ${ }^{3}$ Institute for the Physics and Mathematics of the Universe, University of Tokyo, Kashiwanoha \\ 5-1-5, Kashiwa, Chiba 277-8568, Japan
}

\begin{abstract}
We successfully obtained the first optical spectra of the faint light echoes around Cassiopeia A and Tycho Brahe's supernova remnants (SNRs) with FOCAS and the Subaru Telescope. We conclude that Cas A and Tycho's SN 1572 belong to the Type IIb and normal Type Ia supernovae, respectively. Light echo spectra are important in order to obtain further insight into the supernova explosion mechanism of Tycho's SN 1572: how the Type Ia explosion actually proceeds, and whether accretion occurs from a companion or by the merging of two white dwarfs. The proximity of the SN 1572 remnant has allowed detailed studies, such as the possible identification of the binary companion, and provides a unique opportunity to test theories of the explosion mechanism and the nature of the progenitor. Future light-echo spectra, obtained in different spatial directions of SN 1572, will enable to construct a three-dimensional spectroscopic view of the explosion.
\end{abstract}

Keywords. supernovae: individual (SN 1572, Tycho's supernova)

\section{Introduction}

The supernova of 1572 marked a milestone in the history of science. From his accurate astrometric observations of the SN, Tycho Brahe concluded that the "new star" in the constellation of Cassiopeia must be located far beyond the Moon. This contradiction to the Aristotelian concept, that a change on the sky can only occur in the sub-lunar regime, ultimately led to abandoning the immutability of the heavens. The exact spectroscopic classification of SN 1572 has since been controversial. Light echoes of an outgoing supernova flash by interstellar dust near the remnant have made it possible to re-observe historical SNe after centuries, and to precisely determine the types of their explosions. Using such light-echoes we have been able to re-observe the same light Tycho saw more than 400 years ago and to spectroscopically analyze it today with the FOCAS instrument.

\section{Results}

The light-echo spectrum of Tycho Brahe's SN 1572 shown in Figure 1 matches well spectra of other core-normal SNe Ia (Krause et al. 2008). We have compared the spectrum of SN 1572 with thermonuclear supernovae of different luminosity. Both sub- and overluminous SNe Ia, such as SN 1991bg and SN 1991T, show characteristic peculiarities in their spectra near maximum light and do not provide a good match to our spectrum of SN 1572: SN 1991T lacked a well-defined Si II 6,355^ absorption feature at maximum light. The lack of Si II absorption in SN $1991 \mathrm{~T}$ is visible as an imprint in the time-averaged 


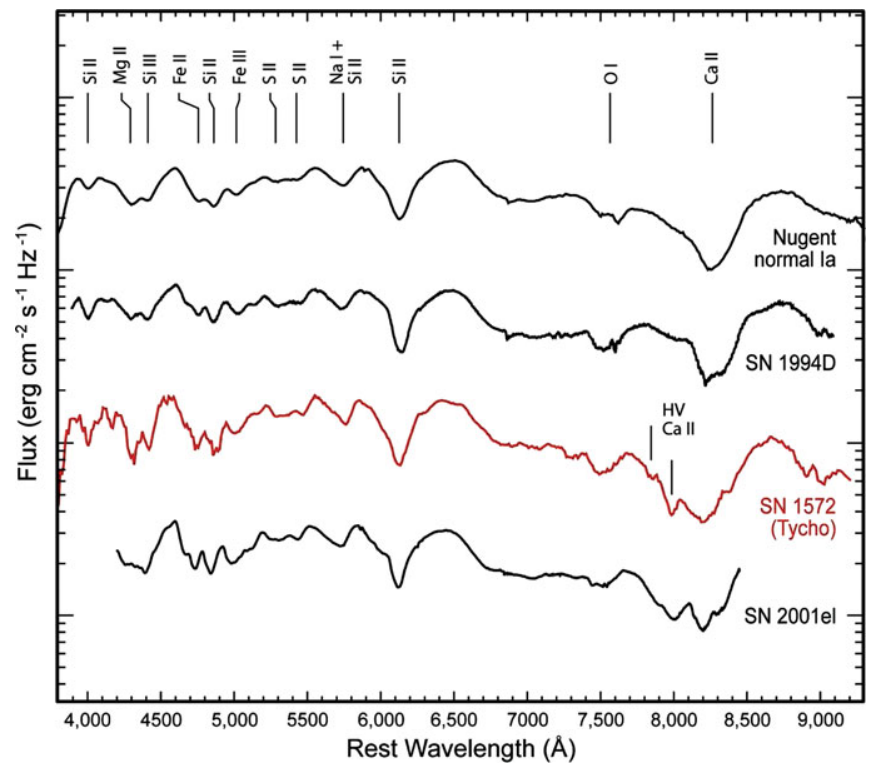

Figure 1. Light-echo spectrum of SN 1572 in comparison with other core-normal SNe Ia.

spectrum, and is different from the strong Si II feature in our spectrum of SN 1572. The class of subluminous objects shows a characteristic deep absorption trough at a wavelength of $4,200 \AA$, attributed to Ti II, near maximum light. Such a feature is not seen for SN 1572. An interesting difference between the spectra of SN 1572 and those of normal SNe Ia is a deep absorption feature at $7,980 \AA$ in the vicinity of the Ca II infrared triplet. While normal SNe Ia, including SN 1572, show a photospheric absorption minimum of the Ca II infrared triplet at a velocity of $13,500 \mathrm{~km} \mathrm{~s}^{-1}$, the additional sharp absorption in SN 1572 corresponds to high velocity components of the Ca II triplet at velocities between 20,000 and $30,000 \mathrm{~km} \mathrm{~s}^{-1}$. High velocity (HV) components have been shown to be ubiquitous in early spectra of SNe Ia, however they are often mixed with the photospheric absorption. To our knowledge, a feature as strong as that observed in the SN 1572 spectrum has only occasionally been detected in SNe Ia spectra, e.g. in SN 2001el.

\section{Outlook}

An exciting opportunity is to use light-echo spectra in different spatial directions to construct a 3-dimensional spectroscopic view of the explosion. This will enable us to determine from observations of a single supernova to what extent spectroscopic diversity can be explained by geometry effects. The observed HV Ca feature in the SN 1572 spectrum might indicate an aspherical explosion. A kinematically distinct HV Ca feature has been also suggested for SN 2001el. In the case that the HV Ca II feature is caused by a single clump, no such feature is expected to be observed in different space directions. Alternatively, the HV Ca II feature could be caused by the interaction with the circumstellar disk of the binary progenitor, in which case the feature might be observed also in other directions.

\section{References}

O. Krause, M. Tanaka, T. Usuda, T. Hattori, M. Goto, S. M. Birkmann, \& K. Nomoto, 2008, Nature, 456, 617 